\title{
Urgences
}

\section{Scénarios pour finir}

\section{René Lapierre}

Numéro 23, avril 1989

Lisière du livre

URI : https://id.erudit.org/iderudit/025515ar

DOI : https://doi.org/10.7202/025515ar

Aller au sommaire du numéro

Éditeur(s)

Urgences

ISSN

0226-9554 (imprimé)

1927-3924 (numérique)

Découvrir la revue

Citer cet article

Lapierre, R. (1989). Scénarios pour finir. Urgences, (23), 46-50. https://doi.org/10.7202/025515ar

Ce document est protégé par la loi sur le droit d'auteur. L'utilisation des services d'Érudit (y compris la reproduction) est assujettie à sa politique d'utilisation que vous pouvez consulter en ligne.

https://apropos.erudit.org/fr/usagers/politique-dutilisation/
Cet article est diffusé et préservé par Érudit.

Érudit est un consortium interuniversitaire sans but lucratif composé de l’Université de Montréal, l'Université Laval et l'Université du Québec à Montréal. Il a pour mission la promotion et la valorisation de la recherche. https://www.erudit.org/fr/ 
Mots et mort

La première personne de Pierre Turgeon

Les heures de Fernand Ouellette

La conférence inachevée de Jacques Ferron

et sur Le désarroi de Julien Bigras et Jacques Ferron 


\section{RENÉ LAPIERRE \\ Scénarios pour finir}

Qu'on écrive du point de vue de la mort, que la pratique de la littérature nous amène peu à peu à tout saisir par la fin, ou comme un film projeté contre la nuit, n'inspire pas trop de morbidité.

Pierre Turgeon, «Pour un roman du désastre»

En couverture un photographisme de Tibo représente, audessus de l'ourlet noir d'une vague, un faisceau géant de routes qui s'éparpillent dans une nuit bleutée. Ce qui étonne dès l'abord c'est que ces routes vont à l'envers; plaquées contre le plafond bas du ciel, elles cherchent dirait-on à déborder le cadre, à sortir de la photo. Comme un avion dont le trajet ascendant passerait audessus de nos têtes, et se perdrait en grondant derrière nous.

Nul personnage pourtant n'apparaît à l'intérieur de l'image; à sa place (ou pour mieux dire à la place qu'il aurait peut-être occupée juste avant au ras des flots, à bord d'une petite embarcation), le regard du lecteur hésite, scrute l'horizon grumeleux. En vain: celui-ci se condense en une brume opaque, brouillard laiteux né de l'écume ou s'y abolissant, on ne sait trop. Collés à la route sombre comme aux arêtes d'une voûte, des centaines de véhicules défilent par ailleurs en rangs serrés: vont-ils? viennent-ils? Difficile encore une fois de le déterminer: leurs phares sont rouges comme des feux arrière, accentuant de la sorte l'effet d'inversion de l'image. Dernière chose enfin, le photographisme oblige à interpréter de façon convergente le déplacement des véhicules: en sorte que, qu'ils s'éloignent ou se rapprochent, tous circulent obstinément dans la même direction. L'effet de cette unidirectionnalité est hallucinant: on croirait, de deux choses l'une, voir émerger un monstrueux corail noir (gorgone, méduse de bitume) ou encore, comme dans un visionnement à rebours, voir l'univers entier se rétracter et disparaître dans un effrayant remous, un vortex abouché au néant.

Fragile, difficilement lisible sur le bleu nuit du fond, le titre se détache à peine: la première personne, sans capitale, apparaît en lettres corail, du même rouge que les énigmatiques feux des automobiles. Semblables à des yeux de chat, épinglés sur l'asphalte comme des points au laser, ceux-ci ont d'ailleurs l'air de chercher à établir avec les trois mots une sorte de connivence, un accord froid 
de quartz, une impersonnelle harmonie cathodique: Ia première personne s'affiche en demeurant en caractères de type E13B, couramment associés aux ordinateurs et aux gadgets électroniques.

L'informatique, la ville, la mer: ces trois éléments du graphisme de couverture orientent la perspective titrale dans un sens particulier, placé comme un point originel entre la vie et la mort, à la frontière du Commencement et de la Fin. Et le livre en effet raconte l'histoire d'un programmateur de la S.Q. (dont le nom "véritable» ne nous est pas donné) qui décide un jour de disparaître civiquement et de renaître aux États-Unis sous une identité nouvelle, qu'il aura lui-même insérée au préalable dans les archives électroniques de la police. La première personne dont il s'agit est donc à entendre à la fois comme instance pronominale (je) et comme effacement de cette instance, ambiguïté que renforcent opportunément l'intitulé des chapitres liminaires (1/Désertion; 12/Désertion (2)) et l'incipit: «Demain je pars pour toujours d'ici.»

À y regarder de plus près, d'ailleurs, c'est l'appareil signifiant tout entier qui est placé sous le signe de cette ambiguïté qui le fonde. Non pas être ou ne pas être, mais plutôt être et ne pas être, mourir et vivre: être et néant. Tout le système présentatif du livre est à la fois affirmatif et négatif, fondation et dérision de l'existence. Le même enjeu se déroule ainsi successivement en plusieurs lieux:

1) d'abord sur la couverture même, entre l'apparition énigmatique du titre (en rouge) et l'assertion éditoriale rassurante (en jaune clair) des noms d'auteur, d'éditeur et de collection. Le même équilibre chromatique et typographique est comme il se doit reproduit sur l'épine, avec inversion toutefois des positions du nom d'auteur et du titre du roman: comme si le chiasme trahissait une autre fois l'indifférence du nom plutôt que l'individuation, et substituait au spécifique (moi, Pierre Turgeon, auteur) le générique (la première personne, n'importe laquelle: le je-miroir de celui qui énonce, qui écrit ou qui lit) ${ }^{1}$;

2) ensuite, entre le recto et le verso de la couverture, où cette fois c'est la photo de l'auteur qui paraît, sévère et barbu comme une sorte d'Hadès, encadrée d'un filet rouge rappelant la couleur du titre et bordant comme de néant la pièce d'identité; 
3) enfin, à l'intérieur même du livre, (passé la page de garde, le rappel des autres publications de l'auteur, la page de titre et la notice relative au copyright) entre la dédicace du livre: «À mon fils François» et l'épigraphe: "La vie est un voyage en automobile." - Jim Morrison.

Dans tous ces lieux se reformule le même enjeu, la même tension entre être et disparaître, (s')énoncer et (s')effacer; jusqu'à ce que le texte atteigne le point de rupture, ou plus exactement le point de fusion de ses contradictions: «À l'approche du zéro absolu, toute matière devient cristalline ou au contraire superfluide. Après le dernier soleil, l'univers ressemblera à un flocon» (p. 29).

En plusieurs endroits du livre se présenteront d'ailleurs des énoncés du même genre, qui laisseront s'amincir la pellicule de l'histoire jusqu'à faire paraître au-dessous d'elle la vérité vide, le creux du récit: "Le vide se trouve derrière, au creux, devant les apparences [...]. Je ne veux surtout pas réfléchir. Penser, c'est poser la mort devant soi, et faire un choix" (p. 85).

Alors écrire, aussi bien. Il y a du Blanchot dans cette formule, qui cherche l'écriture et la pensée du côté de la mort, et qui opte pour elle dans une recherche qui se préoccupe de «descendre [...] jusqu'à un point où ne semble parler que la neutralité impersonnelle ${ }^{2}$. Et que dit alors cette voix blanche, ce neutre équilibre du roman entre le je ancien de l'énonciation et le néant promis? Rien d'autre que cette attente du «zéro absolu», cette non-consommation des choses, du réel et de l'identité: "Je me sens comme dans une gare, à attendre un train qui ne viendra pas: c'est la réalité qui partira, très ponctuellement, me laissant en rade sur le quai, mes valises à la main. Je ne pourrai jamais la rattraper" (p. 85). Et ailleurs: «La sagesse suprême, c'est regarder le monde comme un message publicitaire (p. 17) ${ }^{3}$.

Quelque temps après sa parution, La première personne s'est mérité le Prix du Gouverneur général - section roman pour l'année 1980. Il est amusant de constater que pour l'occasion l'éditeur ne s'est pas contenté de renouveler le tirage du livre, mais qu'il en a complètement modifié la présentation visuelle. Sur un fond maintenant argenté, le photographisme de Tibo a fait place à la reproduction autorisée d'un détail d'une toile d'Alex Colville, Pacific, exécutée en 1967. Deux bandeaux argentés, bordés à l'intérieur d'un trait ocre, encadrent en haut et en bas de la couverture (recto-verso) l'illustration choisie, qui réintègre ainsi sagement la maquette originellement conçue par Roland Giguère pour la 
collection «Prose entière». Sur le bandeau supérieur, bien sûr, la mention «Prix du gouverneur général» (à l'encre noire) surmonte le nom de l'auteur (encre bleue, sans capitales) et le titre du roman (désormais en rouge orangé et en caractères classiques - Plantin ou Times). Le bandeau inférieur pour sa part agrandit la signature éditoriale ("Quinze/prose entière") et lui redonne la dimension qu'elle a partout ailleurs sur les autres couvertures de la collection. Le même ordre est retenu (nom de l'auteur, titre, nom de l'éditeur, traits ocres continus) sans aucune fantaisie, pour l'épine. Au verso, enfin, la photo de l'auteur, rasé de frais et vaguement souriant, est bordée d'un filet noir. Les textes de présentation n'ont pas varié.

Dans l'ensemble la nouvelle présentation est plus équilibrée, plus sobre, mais aussi plus banale. On n'y retrouve plus, de l'illustration dantesque de Tibo, que l'ourlet d'une vague bleue qui vient mourir en contrebas, aux pieds d'un homme qui contemple la mer appuyé, torse nu, au chambranle d'une porte. Dans son dos, posée de travers sur une table de drapier, une arme automatique occupe l'avant-plan de l'image.

En gros, l'utilisation qui est faite ici de l'illustration de Colville détend et déproblématise la perspective titrale du roman, qui ne suggère plus sous sa nouvelle enveloppe que le polar traditionnel, pour ne pas dire le cliché du polar ${ }^{4}$. Le changement de couverture révèle ainsi un net déplacement de la position de lecture du roman: dans le premier cas (Tibo) quelque chose est en train de se produire, nous surprenons le monde dans son chavirement. L'événement est en cours, nous assistons à une représentation en énergie du sens: renversement et consommation du je, ensevelissement de l'instance énonciative dans un univers désormais étranger.

Dans l'autre cas (Colville), curieusement, la représentation bascule dans l'après-coup; elle énonce de façon maintenant statique, dans une sorte d'épuisement désabusé, un conflit qui n'est pas résolu autant que dissout, bémolisé par le recours à la feinte indolence du détective classique, du privé de série noire. Représentation non plus en énergie mais en inertie du changement, de la crise du je qu'illustrent passivement cette fois la silhouette acéphale du "héros» et l'écume de la vague roulant en contrebas (pâle, bleutée, se mourant, et non plus violacée, profonde et déferlante). Or on se rappelle que le roman est écrit au présent, temps précaire et problématique qui entre d'autant plus fortement en collision, dans la seconde édition, avec le cadre graphique qui lui est donné. 
Le cadre en effet signale le livre - la collection - comme moyen, intention de colliger; il identifie, ordonne, et même antériorise: il procure à l'illustration qu'il entoure quelque chose de définitif, un fini qu'elle n'a pas autrement et qui désormais la rend apte non pas seulement à figurer mais encore à fixer, à circonscrire, à résumer. Le photographisme de la première édition semble irréductiblement problématique par rapport à l'édition primée du roman, cadrée et régularisée en fonction d'une position lectorale plus classique, d'un acte de lecture mis à distance, à l'abri de l'acte d'énonciation, et échappant par là même au péril qu'il introduit: «Demain je pars pour toujours d'ici» (p. 13).

Y a-t-il lieu de conclure? II me semble que non. Conclure, ce serait refermer le livre, trancher peut-être en affirmant le bon droit d'une lecture et l'errement, le tort d'une autre. Or, si les lectures divergent, elles ne s'excluent pourtant pas: loin de référer en dernière analyse à un certain contenu (par rapport auquel une lecture serait reçue comme "vraie» ou «fausse», «bonne» ou "mauvaise») cette double présentation du roman offre en fait une lecture de la lecture, une image du récit et du je se distanciant de lui-même et se reprenant (se recueillant, si l'on veut) dans une tradition, sinon à l'intérieur d'un code. Nous passons ainsi de la représentation d'une genèse (voir le bouleversement, le sens dessus-dessous, le conflit de la phrase inaugurale) à la confirmation d'un genre, celui du roman policier; la lecture génésique met l'accent sur la spectaculaire mort-et-naissance du récit (mort de la première personne et naissance du héros) alors que la lecture générique suggère plutôt le morne monologue du détective, l'imperturbable soliloque du privé dans la nuit.

1. Quelques semaines plus tard un second tirage, coïncidant avec l'attribution au roman du Prix du Gouverneur général, renversait de nouveau cet ordre en le conformant cette fois-là à l'usage de la collection "Prose entière» et à la maquette qui lui était ordinairement réservée. Nous y reviendrons un peu plus loin.

2. Maurice Blanchot, Le livre à venir, Paris, Gallimard, 1959, p. 293.

3. Dans un article récemment publié, où Turgeon expose brièvement sa conception de l'écriture et du roman, on peut lire: «On écrit donc dans le suspens du néant [...] Rien n'illustre mieux cette précaire condition que les vanitas calvinistes qui montrent un crâne posé sur un testament, au milieu d'une pile de livres: l'oeuvre comme legs que ne liront peut-être que ces orbites vides et osseuses.» («Pour un roman du désastre», Liberté, Montréal, no 180, décembre 1988, p. 86.)

Et plus haut: «Je n'ai jamais écrit qu'acculé au désastre». Idem, p. 85.

4. Comme pour le confirmer, un éditeur français (Club France Loisirs, Paris) reprenait en 1985 l'illustration de Colville sur la jaquette du roman Les vrais durs ne dansent pas de Norman Mailer. La reproduction, non autorisée sans doute, était cette fois-là un calque grossier de la toile de Colville. 